\title{
Plasma Activation as a Powerful Tool for Selective Modification of Cellulose Fibers towards Biomedical Applications
}

\author{
Olivia Mauger ${ }^{1}$, Sophia Westphal ${ }^{1}$, Stefanie Klöpzig ${ }^{1}$, Anne Krüger-Genge ${ }^{1}$, Werner Müller ${ }^{2}$, \\ Joachim Storsberg ${ }^{1}$ and Jörg Bohrisch ${ }^{1, *}$ \\ 1 Department of Healthcare, Biomaterials \& Cosmeceuticals, Fraunhofer-Institute for Applied Polymer \\ Research (IAP), 14476 Potsdam, Germany; olivia.mauger@iap.fraunhofer.de (O.M.); \\ sophia.westphal@iap.fraunhofer.de (S.W.); stefanie.kloepzig@iap.fraunhofer.de (S.K.); \\ anne.krueger-genge@iap.fraunhofer.de (A.K.-G.); joachim.storsberg@iap.fraunhofer.de (J.S.) \\ 2 QUESTALPHA GmbH \& Co. KG, 35713 Eschenburg, Germany; Werner.Mueller@questalpha.com \\ * Correspondence: joerg.bohrisch@iap.fraunhofer.de
}

Received: 2 October 2020; Accepted: 10 November 2020; Published: 16 November 2020

\begin{abstract}
Cellulosic substrates are known for their biocompatibility, non-cytotoxicity, hypoallergenicity and sterilizability. It is therefore desirable to have a bundle of methods to equip them with tailored properties such as affinity profiles for various applications. In the case of highly swelling materials such as cellulose sponges, "dry" functionalization using plasma activation is the method of choice. The purpose of the study was to adapt low-pressure plasma technology for targeted cellulose modification. Using plasma (pre-) treatment combined with gaseous reactants like $\mathrm{O}_{2}$, ethylene oxide or silane, three different cellulose modifications were obtained and characterized by X-ray photoelectron spectroscopy (XPS) and Fourier transform infrared spectroscopy (FTIR). Swelling measurements and bacterial adhesion tests revealed distinctive material properties compared to educt. The development of these non-aqueous methods demonstrated an effective procedural route towards modified cellulosic materials for usage in wound dressing, micro patterned assays or bacterial filtration.
\end{abstract}

Keywords: low-pressure plasma; plasma activation; regenerated cellulose; bacterial affinity; silanation

\section{Introduction}

Cellulose is the most abundant biopolymer in the world and occurs in all plants.

It is an important building block of the biosphere and could, as a sustainable material, replace non-renewable synthetic polymers [1]. Cellulose-based new materials have fascinating properties and have been frequently used in the life sciences and engineering. So it has long been used in a variety of medical applications ranging from cotton for hemostatic wound dressings to sutures and renal dialysis membranes [2]. The main target of this research was the non-aqueous modification of cellulose-based material consisting of a medical grade sponge made of natural cotton and regenerated cellulose. So the range of applications could be widened towards use in water and sewage treatment, wound dressings and areas where in general the control of bacterial attachments is desirable.

For highly swelling materials such as "cellulose sponges", most reactions in aqueous solvents do not work properly due to the competition between water and cellulosic hydroxyl groups. Therefore, "dry" functionalization using plasma activation is the method of choice.

For example, plasma treatment of cellulose is used for improving adhesion properties of fibers forming a biocomposite with starch [3]. Ning et al. reported a complete hydrophobization of cotton fabric through reactions with hexamethyldisiloxane during plasma activation [4]. Recently, 
a plasma-assisted hydrophobization of a bacterial cellulose membrane using trichlorosilane was reported [5].

In order to preserve the structure and shape of the spongy material, the modification was achieved via plasma technology supported by the use of oxygen and nitrogen as process gases. The aim of the studies was the generation of three different fiber surfaces, hydrophilic but uncharged, slightly charged and a hydrophobic one. Therefore, $\mathrm{N}_{2}$ as a process gas was chosen for the first sample, and $\mathrm{O}_{2}$ for the latter ones.

The characterization of the structure and the surface properties of the modified material was performed by microscopy, attenuated total reflection-FTIR (ATR-FTIR) and X-ray photoelectron spectroscopy (XPS). The attained properties were demonstrated by bacterial absorption, contact angle measurement and swelling tests. The modification experiments were performed on small sponge discs characterized by a diameter of $12 \mathrm{~mm}$ and a grammage of $160 \mathrm{~g} / \mathrm{m}^{2}$.

The cellulose surface modification was planned using low-pressure plasma in order to induce oxygen and nitrogen functionalization and reactive groups on the fiber surface. The experimental conditions were chosen using one set of parameters (except $\mathrm{N}_{2}$ usage for sample KB-1, see Table 1) tested successfully for other hydroxyl containing polymers.

Table 1. Low-pressure plasma parameters using Flecto 10 plasma device.

\begin{tabular}{|c|c|c|c|c|c|}
\hline Sample & $\begin{array}{c}\text { Process } \\
\text { Gas }\end{array}$ & $\begin{array}{c}\text { Gas Flow/Flow } \\
\text { Time }\end{array}$ & Pressure (bar) & $\begin{array}{l}\text { Chamber Vacuum } \\
\text { (mbar) }\end{array}$ & $\begin{array}{c}\text { Plasma } \\
\text { Power (W) }\end{array}$ \\
\hline KB-1 & $\mathrm{N}_{2}$ & $\begin{array}{l}5 \mathrm{sccm} \\
2 \mathrm{~min}\end{array}$ & $\max .1 .5$ & 0.20 & 150 \\
\hline KB-7 & $\mathrm{O}_{2}$ & $\begin{array}{l}5 \mathrm{sccm} \\
2 \mathrm{~min}\end{array}$ & $\max .1 .5$ & 0.20 & 150 \\
\hline KB-9 & $\mathrm{O}_{2}$ & $\begin{array}{l}5 \mathrm{sccm} \\
2 \mathrm{~min}\end{array}$ & $\max .1 .5$ & 0.20 & 150 \\
\hline
\end{tabular}

The main aim was to explore plasma activation of spongy cellulose material to obtain new material properties via gas phase modification using silane and ethylene oxide with subsequent bacterial adsorption studies.

\section{Materials and Methods}

\subsection{Sample Preparation}

Sugi ${ }^{\circledR}$ cellulose sponges (by courtesy of the company QUESTALPHA GmbH \& Co. KG, (Eschenburg, Germany) are made of regenerated cellulose and cotton. After washing and drying the native sponge material, discs with a diameter of $12 \mathrm{~mm}$ were die-cut and packed for further processing (e.g., plasma treatment). Sugi ${ }^{\circledR}$ sponge is biocompatible, lint-free and offers a high stability in a variety of chemicals and solutions. It has a very high and fast absorption capacity for water ranging from $1200-2100 \%$ depending on the material specification.

Plasma treatment was performed using a low-pressure plasma oven (Flecto 10, from Plasma Technology GmbH, Herrenberg-Gülstein, Germany; max. power $300 \mathrm{~W}$ at 40-100 kHz). Table 1 gives an overview of the three plasma treatments of the cellulose discs.

$\mathrm{N}_{2}$ was taken from a lab line and $\mathrm{O}_{2}$ from a $5 \mathrm{~L} \mathrm{O}_{2}$ concentrator, Compact 525 from DeVilbiss Healthcare $\mathrm{GmbH}$, Mannheim, Germany.

The ethylene oxide (EO) treatment system consisted of a $0.5 \mathrm{~L}$ steel autoclave connected on one side to a $0.3 \mathrm{~L}$ EO steel cylinder (purity 99.9\%) purchased from Linde AG (Leuna, Germany) and on the other side to an outlet leading into two $500 \mathrm{~mL}$ gas-washing bottles filled with $2.5 \%$ aqueous sulfuric acid as a neutralizing agent. EO pressure was $1.2-1.3$ bar, leading to a concentration of $2.5 \mathrm{mg} / \mathrm{mL}$ EO in the reactor (about $0.5 \mathrm{~g}$ in $200 \mathrm{~mL}$ volume). 
Silanation required a desiccator with a $700 \mathrm{~mL}$ useable volume as well as a drying cabinet. Methyldimethoxysilane (MDMS, CAS-Nr.: 16881-77-9) was purchased from abcr GmbH Karlsruhe, Germany.

\subsection{Cellulose Treatment}

KB-1: After plasma activation with $\mathrm{N}_{2}$ (gas flow: $5 \mathrm{sccm}$ for $2 \mathrm{~min}$ ), the cellulose discs were treated in a steel autoclave with $\mathrm{EO}$ for $10 \mathrm{~min}$.

KB-7: The plasma treatment of this series was a stand-alone trial where the process gas $\mathrm{O}_{2}$ directly affected the cellulose fibers.

KB-9: Prior to silanation, the treatment of the discs started with a plasma activation using $\mathrm{O}_{2}$ as a process gas. Silanation itself occurred by exposure of the discs to MDMS in a desiccator. MDMS was chosen for chemical vapor deposition (cvd), which was performed under slightly reduced pressure and mild temperatures due to its low boiling point of $61^{\circ} \mathrm{C}$. Typically, a small amount of MDMS $(0.4 \mathrm{~g})$ was poured in a crystallizing dish with a diameter of $4 \mathrm{~cm}$. The discs were placed immediately after plasma activation in a Petri dish on the porcelain desiccator plate above the MDMS filled dish, leading to a concentration of about $0.6 \mathrm{~g} / \mathrm{L}$ in the desiccator. After evacuation of the desiccator to about $400 \mathrm{mbar}$, the vapor deposition reaction took place in a drying cabinet held at $40{ }^{\circ} \mathrm{C}$ for $30 \mathrm{~min}$.

\subsection{Sample Characterization}

The equipment for XPS was the Kratos Axis 165 XPS device from Kratos Analytical Ltd., Manchester, U.K. (Sampling depth $<\sim 7 \mathrm{~nm}$, sensitivity: $0.02-0.5$ at \%). This method enables the investigation of surfaces and delivers quantitative information on their elemental composition. Electrons are detached from the atomic shell are as they are exposed to roentgen radiation and the measured energy specifically characterizes elements and chemical bonding states.

ATR-FTIR was performed with an FT-IR Nexus ${ }^{\circledR}$ spectrometer from Thermo Scientific ${ }^{\mathrm{TM}}$ Nicolet $^{\mathrm{TM}}$ (Berlin, Germany) equipped with Smart Endurance ATR accessory with a sapphire prism with a diameter of $1.64 \mathrm{~mm}$. This infrared spectroscopy technique allows for surface analysis with a penetration depth in the micron range.

Contact angle (CA) analysis was performed with an OCA 15 from DataPhysics Instruments, Filderstadt, Germany. This method gives valuable insights into the hydrophilicity/hydrophobicity of samples by calculation of the angle built by interaction of a liquid —-here, deionized water-with a solid surface. Hydrophobic materials exhibit high CA values; in contrast, liquid spreads on hydrophilic ones and the CA values are low.

\subsection{Swelling Test}

After accurate weighing of each disc, they were soaked in distilled water for $1 \mathrm{~h}$ and then weighed again. The average difference of at least 10 discs was taken as the swelling value.

\subsection{Antibacterial Test}

Cultivation of Escherichia coli:

An overnight culture of Escherichia coli (E. coli) (DSM 116, DSMZ, Braunschweig, Germany) in nutrient broth (Carl Roth, Karlsruhe, Germany) was used to inoculate fresh sterile nutrient broth with E. coli bacteria. The E. coli suspension was incubated on an orbital shaker at $110 \mathrm{rpm}$ and $37^{\circ} \mathrm{C}$.

Filtration and quantification of E. coli:

A defined amount of $E$. coli suspension was filtrated using a manual vacuum syringe with filter (QUESTALPHA) and Stericup ${ }^{\circledR}$ equipment (Merck, Darmstadt, Germany). A quantification of E. coli before and after filtration was performed by plating out $100 \mu \mathrm{L}$ of the suspension on nutrient agar (Carl Roth, Karlsruhe, Germany), overnight incubation at $37^{\circ} \mathrm{C}$ and quantification of colony-forming units (CFUs). 


\section{Results}

\subsection{Chemical Modifications}

The modification method leading to the introduction of polyethylene glycol (PEG)-moieties (labeled as KB-1) consisted of two steps. First, the plasma activation of the cellulose in an $\mathrm{N}_{2}$ atmosphere created a mixture of additional N-containing starting groups for covalent grafting of $\mathrm{EO}$ (see Table 2). The coupling of the EO molecules on its surface and the EO polymerization, as well as the chemical crosslinking, were promoted by a combination of radical formation, $\mathrm{H}$-abstraction and nucleophilic attacks on EO carbon. Plasma treatment of cellulose with $\mathrm{O}_{2}$ only led to an increase in O-functional groups (such as carboxylic, aldehyde or peroxide due to post-plasma reactions [6]) on the fiber surface (labeled as KB-7). In an aqueous environment, this usually leads to negatively charged fibers [7].

Table 2. Surface atom distributions by XPS analysis.

\begin{tabular}{ccccc}
\hline Sample & C (at \%) & N (at \%) & O (at \%) & Si (at \%) \\
\hline KB-0 & $65.2 \pm 0.3$ & & $34.8 \pm 0.2$ & \\
KB-1 & $55.9 \pm 0.3$ & $4.4 \pm 0.02$ & $39.5 \pm 0.2$ & \\
KB-7 & $56.7 \pm 0.3$ & & $42.6 \pm 0.2$ & \\
KB-9 & $34.6 \pm 0.2$ & & $34.0 \pm 0.2$ & $31.3 \pm 0.2$ \\
\hline
\end{tabular}

Silanation was achieved via chemical cvd after activation by plasma [8,9].

As organosilanes possess a hydrolytically sensitive center, the silane molecules react efficiently with hydroxyl-containing substrates; this was exploited for the third modification (labeled as KB-9).

\subsection{Analyses}

The surface analysis by XPS revealed remarkable differences in atom distribution, as shown in Scheme 1. Untreated and clean cellulose typically exhibits two peaks in C1s XPS originating from alcohols, ethers (C-O, $286.7 \mathrm{eV}$ ) and acetal groups (O-C-O, $288.3 \mathrm{eV})$. However, as this complex fibrous structure is often altered by in situ degradation, a signal at $285.0 \mathrm{eV}$ corresponding to a C-C bond peak is mostly present, due to air exposure and, hence, contamination of the fiber surface.

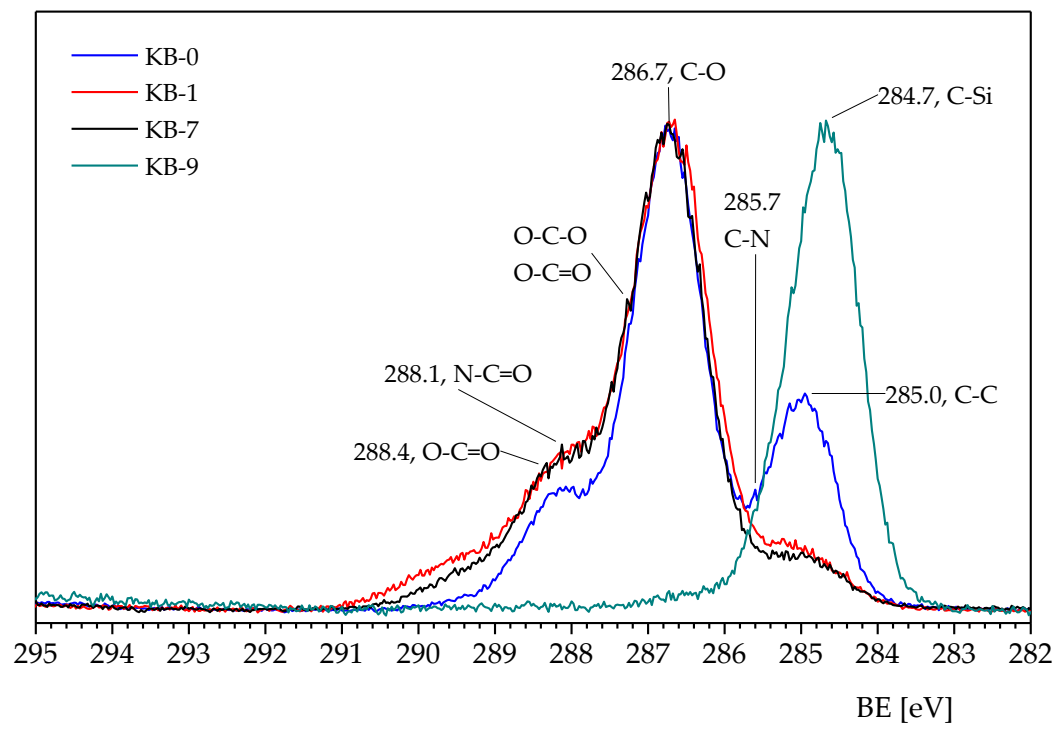

Scheme 1. X-ray photoelectron spectroscopy (XPS) imaging of C 1s spectra of KB-0, -1, -7 and -9. 
The XPS spectrum of untreated cellulose material, KB-0, reflected these features with a major C-O peak and a significant C-C peak. It further exhibited smaller peaks due to C-N (C-N: $285.7 \mathrm{eV}$ ), $\mathrm{N}-\mathrm{C}-\mathrm{O}$ and $\mathrm{C}-\mathrm{O}(288.1,288.4 \mathrm{eV})$ bonds probably also due to residues and contaminations.

XPS of KB-1 and KB-7 again exhibited the strong C-O signals at $286.7 \mathrm{eV}$ together with the N-C-O and $\mathrm{O}-\mathrm{C}=\mathrm{O}$ peaks whose intensity increased. A closer examination of KB-1 via N1s XPS attested the increase in $\mathrm{C}-\mathrm{N}$ bonds on the sample surface.

Interestingly, the C-C peak after plasma treatment was absent in all samples. This showed the substitution of $\mathrm{C}-\mathrm{C}$ bonds by new functional groups.

The C1s XPS of KB-9 showed a completely different surface from the original cellulose outer shell: at a depth of about $7 \mathrm{~nm}$, it seemed to be totally covered by the silane coating (C-Si: $284.7 \mathrm{eV}$ ).

The elemental composition results are listed in Table 2. The quantitative analysis of the layer compounds was achieved by measurements of the peak areas and calculation with the use of standard samples, the method applied in the Kratos spectrometers to determine the atomic sensitivity factors. The detection limits for XPS range from 0.02 to 0.5 atomic percent (at \%).

The N percentage in KB-1 was due to $\mathrm{N}_{2}$-plasma activation, $\mathrm{C}$ was reduced as expected. In KB-7, a significant increase in $\mathrm{O}$ percentage after $\mathrm{O}_{2}$-plasma was detected. $\mathrm{KB}-9$ surface was strongly enriched with silicon.

This was also confirmed by ATR-FTIR spectra of treated samples compared to blank KB-0 (Figure 1).

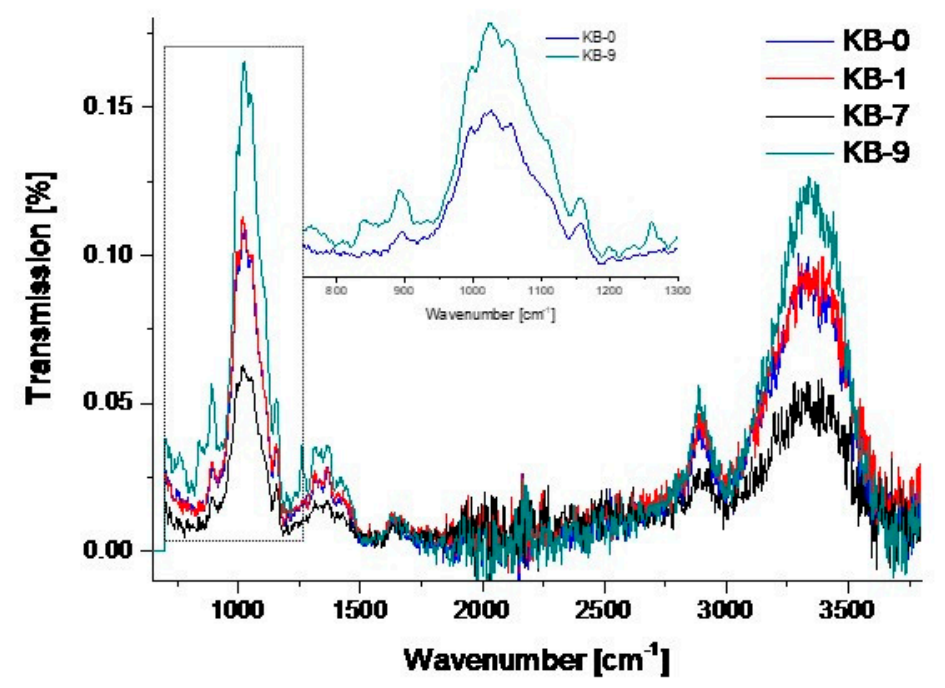

Figure 1. Attenuated total reflection Fourier transform infrared spectroscopy (ATR-FTIR) spectra of KB-0, $-1,-7$ and -9 with an enlarged segment (rectangle: $750-1300 \mathrm{~cm}^{-1}$ ) of spectra of KB-0 and -9 .

Silanized KB-9 samples exhibited particular changes in their spectra by the emergence of Si-C and Si-O-Si stretching vibrations and symmetric and asymmetric $\mathrm{Si}-\mathrm{CH}_{3}$ rocking vibrations, as well as deformation vibrations: $767\left(v_{\mathrm{s}} \mathrm{Si}-\mathrm{CH}_{3}\right), 835\left(\gamma_{\mathrm{s}} \mathrm{Si}-\mathrm{CH}_{3}\right), 893\left(\gamma_{\mathrm{as}} \mathrm{Si}-\mathrm{CH}_{3}\right), 1028\left(v_{\mathrm{s}} \mathrm{Si}-\mathrm{O}-\mathrm{Si}\right)$, $1259\left(\delta \mathrm{Si}-\mathrm{CH}_{3}\right), 2966\left(v_{\text {as }} \mathrm{Si}-\mathrm{CH}_{3}\right) \mathrm{cm}^{-1}$.

The contact angle of the KB-9 sample amounted to $132.5^{\circ}$ (stable for more than an hour) on average (see Figure 2), providing very good evidence of the hydrophobization of the material. All other samples absorbed the water immediately; no contact angle could be detected.

Swelling tests were performed in water with the treated cellulose discs and compared to the pristine material KB-0. The water intake of the material significantly reflected the changes of the fiber surface properties (Table 3). 

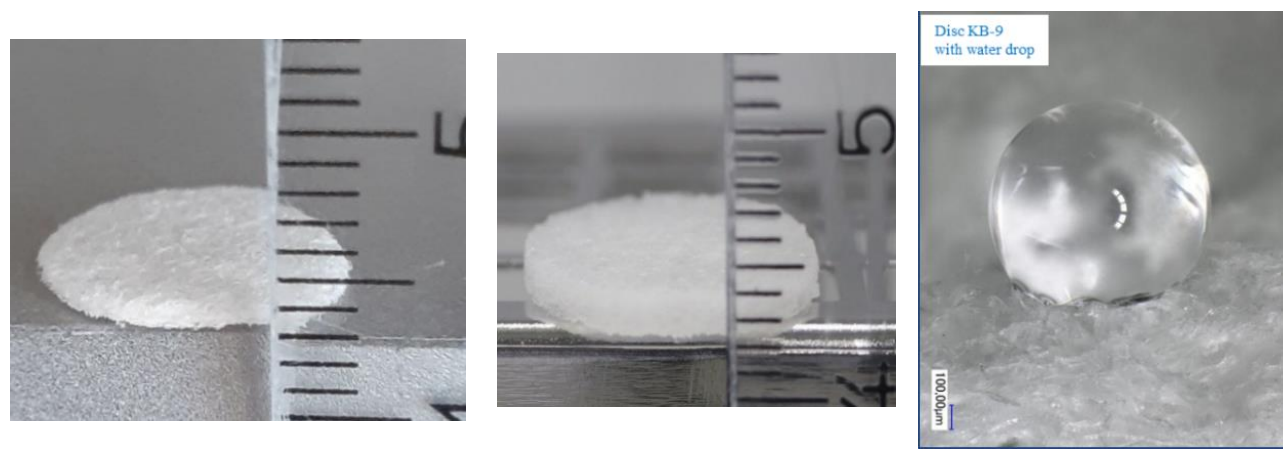

Figure 2. Left: dry Sugi ${ }^{\circledR}$ sponge (KB-0), middle: water-swollen KB-0 with highest water uptake, right: water drop on KB-9.

Table 3. Swelling data of samples KB-0, $-1,-7$ and -9 (average dry weight: $0.02 \mathrm{~g}$ ).

\begin{tabular}{ccc}
\hline Sample & Weight after Swelling (g) & Number of Samples \\
\hline KB-0 & $0.26 \pm 0.02$ & 16 \\
KB-1 & $0.23 \pm 0.05$ & 10 \\
KB-7 & $0.24 \pm 0.03$ & 10 \\
KB-9 & $0.040 \pm 0.002$ & 61 \\
\hline
\end{tabular}

The water absorption indicated the modification grade of the discs, as it gradually decreased from KB-0, KB-1 and KB-7. The silanized disc KB-9 only absorbed a very small fraction of water compared to the untreated cellulose material, showing strong water repellence due to the remarkable hydrophobization. The swelling capacity therefore was an adequate criterion of the material transformation that enabled a brief insight into the property alterations.

\subsection{Test of E. coli Adsorption}

In order to explore the potential of these modifications for specific filtration purposes, a suspension of $E$. coli in nutrient growing medium was percolated through a vacuum filtration unit using pristine and modified cellulose discs as filter membranes. Passing through the modified cellulose sponge discs KB-7 and KB-9 was followed by the analysis of colony-forming units (CFUs). A reduction of E. coli in the filtrate of modified compared to the untreated material KB-0 was observed. The reduction of CFUs using KB-7 amounted to $51.1 \%$ (CFU value reduction from $14.3 \pm 1.0 \times 10^{9}$ to $7.0 \pm 1.0 \times 10^{9}$ ) and to $22.5 \%$ with KB-9 (CFU value reduction from $12.0 \pm 1.0 \times 10^{9}$ to $9.3 \pm 2.5 \times 10^{9}$ ).

In contrast, the use of the KB-1 disc as a filter for an E. coli suspension did not show any effect ( $\mathrm{CFU}$ value reduction from $8.0 \pm 1.1 \times 10^{9}$ to $7.3 \pm 1.0 \times 10^{9}$ ) so $\mathrm{KB}-1$ was similar to the unmodified KB-0 (Figure 3).

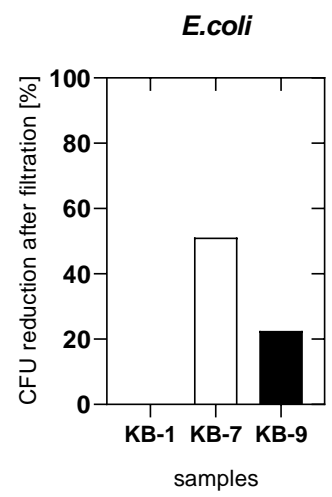

Figure 3. Reduction of $\mathrm{CFU} / \mathrm{mL}$ after filtration through three different cellulose modifications compared to pristine cellulose $\mathrm{KB}-0$ (=0\%). 


\section{Discussion}

Spongy cellulose material is widely used in medical applications as, for example, absorber pads, diagnostic tools and wound care applications. The improvement and tailoring of its set of properties is challenging due to the special morphology, which affords "dry" modification. Therefore, three basic plasma-assisted methods were developed to implement different fiber properties. In order to get insight regarding the principles of interaction with bacteria such as $E$. coli, three generally different modifications were chosen. In detail, uncharged or charged hydrophilic, as well as hydrophobic, material was synthesized. Hydroxyethylation of cellulose is normally carried out as a "wet" method using alkaline conditions [10]. Plasma treatment in a nitrogen atmosphere with the subsequent addition of ethylene oxide led to a nonionic fiber modification with (oligo) ether moieties and some additional nitrogen-containing groups on the surface ( $\mathrm{N}$-content $4.4 \%$, see Table 2 ). This kind of modification is known to display smooth and somewhat inert surfaces with low bacterial adhesion. The hydrophilicity and affinity towards E. coli was similar to unmodified sponge. An increase in oxygen-rich groups (e.g., carboxylic) was generated using an oxygen atmosphere during plasma treatment. The slightly charged modification displayed a high affinity in the E. coli filtration test (Figure 3). This complies with Iwata et al., who stated support for oxygenated groups for improved cell adherence [11]. Therefore, this could be an important feature in the development of special units for bacterial filtration purposes. Finally, silanation using MDMS with plasma pre-treatment resulted in a very hydrophobic material under the chosen conditions. This method separated the activation from vapor deposition on one hand (compare [4]) and avoided the usage of a toxic trichlorosilane (compare [5]) on the other. XPS and FTIR measurement proved the high proportion of silicon on the fiber surface (Table 2, Figure 1). While the water absorption of the species KB-0, -1, and -7 was more than 10 times their own weight, here, only a doubling took place (Table 3$)$. The retention of bacteria ( $20 \%)$ with this uncharged material was remarkable. Hence, a modulation of the hydrophobic properties could advance this cellulose modification to a suitable candidate for special filtration applications.

\section{Conclusions}

The aim of this investigation was the modification of regenerated cellulose-based discs by non-aqueous methods. In order to study the interaction of differently modified (regarding charge and hydrophilicity) cellulose fibers with E. coli, three materials were developed. Both the oxidized (charged, hydrophilic) and the silanized (strongly hydrophobic) ones led to a stronger attachment of E. coli, providing an enormous potential for applications in water and sewage treatment, for example. This points to the fact that the parameters of charge and hydrophobicity play an important role in designing appropriate surfaces for bacterial interaction devices.

In order to prove the broad use of this material, further studies regarding detailed material analysis, cytotoxicity and compatibility with cells are currently underway.

Author Contributions: Conceptualization, J.B., J.S. and W.M.; methodology, J.B., O.M. and A.K.-G.; software, J.B.; validation, J.B., O.M., A.K.-G. and J.S.; formal analysis, J.B.; investigation, J.B., O.M., S.W., S.K. and A.K.-G.; resources, J.B. and O.M.; data curation, J.B. and O.M.; writing-original draft preparation, J.B. and O.M.; writing-review and editing, J.B., J.S. and W.M.; visualization, J.B.; supervision, J.B.; project administration, J.B. All authors have read and agreed to the published version of the manuscript.

Funding: This research received no external funding.

Acknowledgments: The authors would like to thank Karsten Tauber (QUESTALPHA GmbH \& Co. KG, 35713 Eschenburg, Germany) for helpful technical discussions and providing Sugi ${ }^{\circledR}$ samples.

Conflicts of Interest: The authors declare no conflict of interest.

\section{References}

1. Klemm, D.; Heublein, B.; Fink, H.P.; Bohn, A. Cellulose: Fascinating Biopolymer and Sustainable Raw Material. Angew. Chem. Int. Ed. 2005, 44, 3358-3393. [CrossRef] [PubMed] 
2. Hoenich, N. Cellulose for Medical Applications: Past, Present and Future. BioResources 2006, 1, $270-280$. [CrossRef]

3. Fazeli, M.; Florez, R.; Simão, A. Improvement in adhesion of cellulose fibers to the thermoplastic starch matrix by plasma treatment modification. Compos. Part B Eng. 2019, 163, 207-216. [CrossRef]

4. Yang, J.; Pu, Y.; Miao, D.; Ning, X. Fabrication of Durably Superhydrophobic Cotton Fabrics by Atmospheric Pressure Plasma Treatment with a Siloxane Precursor. Polymers 2018, 10, 460. [CrossRef] [PubMed]

5. Leal, S.; Cristelo, C.; Silvestre, S.; Fortunato, E.; Sousa, A.; Alves, A.; Correia, D.M.; Lanceros-Mendez, S.; Gama, M. Hydrophobic modification of bacterial cellulose using oxygen plasma treatment and chemical vapor deposition. Cellulose 2020, 1-14. [CrossRef]

6. De Geyter, N.; Morent, R. Cold plasma surface modification of biodegradable polymer biomaterials. In Biomaterials for Bone Regeneration, 1st ed.; Dubruel, P., Van Vlierberghe, S., Eds.; Woodhead Publishing: Sawston, UK, 2014; pp. 202-224.

7. Tiwari, S.; Bijwe, J. Surface Treatment of Carbon Fibers-A Review. Procedia Technol. 2014, 14, 505-512. [CrossRef]

8. Thakur, M.K.; Gupta, R.K.; Thakur, V.K. Surface modification of cellulose using silane coupling agent. Carbohydr. Polym. 2014, 111, 849-855. [CrossRef] [PubMed]

9. Zhang, F.; Sautter, K.; Larsen, A.M.; Findley, D.A.; Davis, R.C.; Samha, H.; Linford, M.R. Chemical Vapor Deposition of Three Aminosilanes on Silicon Dioxide: Surface Characterization, Stability, Effects of Silane Concentration, and Cyanine Dye Adsorption. Langmuir 2010, 26, 14648-14654. [CrossRef] [PubMed]

10. Zhou, Q.; Zhang, L.; Li, M.; Wu, X.; Cheng, G. Homogeneous hydroxyethylation of cellulose in NaOH/urea aqueous solution. Polym. Bull. 2005, 53, 243-248. [CrossRef]

11. Arima, Y.; Iwata, H. Effect of wettability and surface functional groups on protein adsorption and cell adhesion using well-defined mixed self-assembled monolayers. Biomaterials 2007, 28, 3074-3082. [CrossRef]

Publisher's Note: MDPI stays neutral with regard to jurisdictional claims in published maps and institutional affiliations.

(C) 2020 by the authors. Licensee MDPI, Basel, Switzerland. This article is an open access article distributed under the terms and conditions of the Creative Commons Attribution (CC BY) license (http://creativecommons.org/licenses/by/4.0/). 